\title{
Photonic THz Generation Using Optoelectronic Oscillator driven Optical Frequency Comb Generator
}

\author{
G.K.M.Hasanuzzaman ${ }^{1}$, Haymen Shams ${ }^{2}$, Cyril C. Renaud ${ }^{2}$, John Mitchell ${ }^{2}$ and Stavros Iezekiel ${ }^{1 *}$ \\ ${ }^{1}$ Department of Electrical and Computer Engineering, University of Cyprus, Nicosia 1678, Cyprus \\ ${ }^{2}$ Department of Electrical and Electronic Engineering, University College London, Torrington Place, London, WC1E 7JE, UK \\ *iezekiel@ucy.ac.cy
}

\begin{abstract}
We propose and experimentally demonstrate a photonic THz signal generation technique combining a discrete optoelectronic oscillator (OEO) and optical frequency comb (OFC) generator. Using a microwave photonic filter (MWPF), we generate an electrical oscillation up to $18.36 \mathrm{GHz}$ with a phase noise of $-103 \mathrm{dBc} / \mathrm{Hz}$ at $10 \mathrm{kHz}$ offset frequency. The OEO frequency tunability is obtained by changing the bandwidth of a tunable optical band pass filter (TOBF). This can produce an electrical RF carrier from 6.58 - $18.36 \mathrm{GHz}$. The OEO driven optical comb generates 22 optical comb lines with a frequency spacing of $17.33 \mathrm{GHz}$ covering a bandwidth of $360 \mathrm{GHz}$ within a $20 \mathrm{~dB}$ envelop. By selecting two optical comb lines using a wavelength selective switch (WSS) and beating them in a unitravelling carrier photodiode (UTC-PD), a THz wave is generated at $242.6 \mathrm{GHz}$ with phase noise of $-78 \mathrm{dBc} / \mathrm{Hz}$ at $10 \mathrm{kHz}$ offset frequency. This technique would be a potential for use in $\mathbf{T H z}$ signal generation where it is possible to tune the $\mathrm{THz}$ carrier frequency by tuning the RF carrier generated from the OEO.
\end{abstract}

Keywords-Optical frequency comb, optoelectronic oscillator, microwave photonic filter, phase noise.

\section{INTRODUCTION}

Optical heterodyning is a well-known approach to the photonic generation of millimeter wave (mm-wave) and $\mathrm{THz}$ signals. One simple implementation is the use of a pair of freerunning lasers with a wavelength separation corresponding to the desired RF frequency to be generated, but this approach suffers from frequency and phase fluctuations [1]. In contrast, optical frequency comb (OFC) generators overcome this problem due to their highly phase correlated optical tones with a fixed frequency spacing [2]. By appropriate two comb lines selection, it is possible to generate pure mm-wave and $\mathrm{THz}$ signals [3].

Several techniques have been considered to generate OFCs, such as mode-locked lasers, gain-switched lasers, non-linear optics, and external modulation [4]. Conventionally, an external microwave synthesizer is used to provide the seed tone and to determine the frequency spacing in an external modulation based OFC. However, the external synthesizer can be replaced by an optoelectronic oscillator (OEO) which can generate spectrally pure microwave signals with a very low phase noise level [5]. In an OEO, the phase noise is independent of the oscillation frequency while in close-tocarrier, it depends on the $Q$-factor of the optical storage element (either a long optical fiber or whispering gallery mode resonator) used in the OEO loop. In addition, the white noise floor is limited by the shot noise of the photo-detector [6]. A state-of-the-art OEO provides a low phase noise of -
$163 \mathrm{dBc} / \mathrm{Hz}$ at an offset frequency of $6 \mathrm{kHz}$ (for $10 \mathrm{GHz}$ oscillation carrier) [7]. Therefore, the use of an OEO to drive an OFC generator (either directly or via a self-oscillating approach) for subsequent low-phase noise generation of $\mathrm{mm}$ wave and $\mathrm{THz}$ signals is a promising concept.

In first-generation of OEOs, a microwave bandpass filter was used to determine the oscillation frequency, with additional techniques such as dual optical loop being used to suppress electrical side modes [8]. OEOs based on a microwave photonic filters (MWPF) have shown the capability to overcome the tunability limitation of their electrical filter counterparts [9], [10]. In [9], a tunable OEO based on stimulated Brillouin scattering (SBS) was proposed to achieve a carrier with a tunable frequency from DC - $60 \mathrm{GHz}$, while in [10] a tunable OEO $(3-28 \mathrm{GHz})$ was implemented in which two optical phase modulators (PMs) were cascaded with a phase shifted fiber Bragg grating (PS-FBG). A relatively simple technique was also reported in [11] to achieve a tunable frequency range from $4.74 \mathrm{GHz}-38.8 \mathrm{GHz}$ based on using an optical PM and a tunable optical bandpass filter (TOBF), where the tunability was realized by changing the optical bandwidth of TOBF. Later, a modified version of [11] was reported for tuning range up to $57.7 \mathrm{GHz}$ [12].

In our earlier work, we experimentally generated a mmwave signal from a self-oscillating optical frequency comb by using a dual drive Mach-Zehnder modulator (DD-MZM), and a conventional electrical band pass filter in the feedback loop [13], [14]. A $95 \mathrm{GHz}$ mm-wave was generated using this method and a radio over fiber link was implemented to transmit wireless signals modulated with a standard LTE advanced signal. However, this proposed system still lacks of the frequency tunability of the OEO, and thus the generated mmwave at a fixed carrier.

In this paper, we propose and experimentally demonstrate the photonic generation of $\mathrm{THz}$ signal based on a frequency tunable dual-loop $\mathrm{OEO}$, and an optical frequency comb generator (OFCG). We use a MWPF-based approach to achieve the OEO frequency tunability $(6.58-18.36 \mathrm{GHz})$, where a PM and TOBF function together as a MWPF [12]. The system provides the opportunity to generate a tunable low phase noise $\mathrm{THz}$ signal without requiring any reference RF input. The proposed system generates a seed RF signal from $\mathrm{OEO}$ at $17.33 \mathrm{GHz}$ and a phase noise level of $-103 \mathrm{dBc} / \mathrm{Hz}$ at $10 \mathrm{kHz}$ offset frequency. This is used as a driving electrical signal to generate 22 optical comb lines, and covering a range of $360 \mathrm{GHz}$ within a $20 \mathrm{~dB}$ envelop. By selecting two optical comb lines, and beating them in a uni-travelling carrier

This project has received funding from the European Union's Horizon 2020 research and innovation programme under the Marie Skłodowska-Curie grant agreement No. 642355 . 


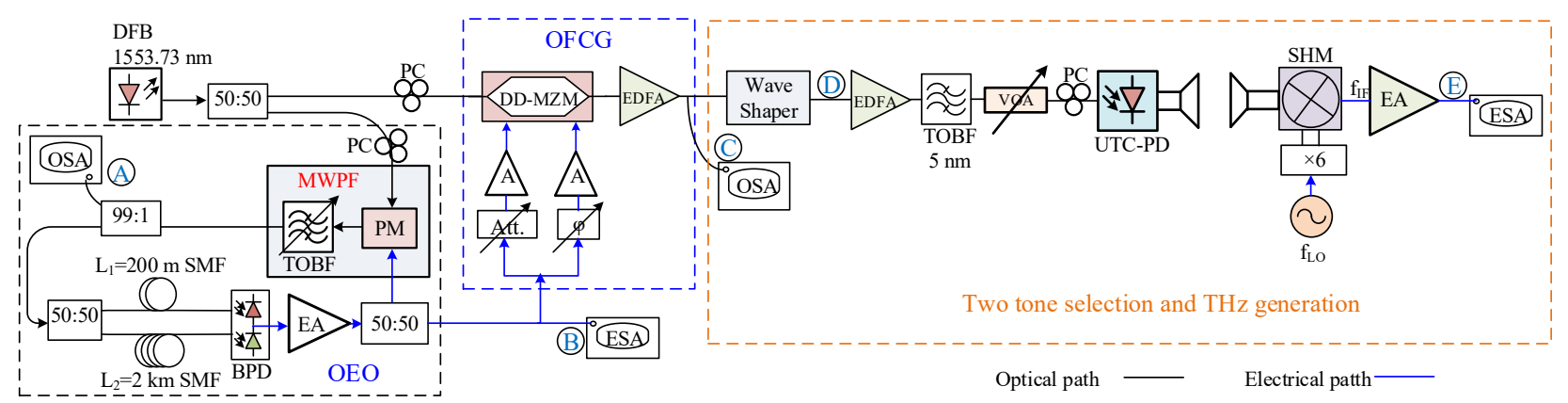

Fig.1. Experimental arrangement of the $\mathrm{OEO}$ driven $\mathrm{OFC}$ generation and $\mathrm{THz}$ wave generation

photodiode (UTC-PD), we generate a THz signal at $242.6 \mathrm{GHz}$. The measured phase noise (after down conversion procedure) is $-78 \mathrm{dBc} / \mathrm{Hz}$ at $10 \mathrm{kHz}$ offset frequency.

\section{EXPERIMENTAL ARRANGEMENT}

The experimental arrangement of the proposed concept is shown in Fig. 1. It consists of the OEO section, OFC generation section, and two optical tones filtering and $\mathrm{THz}$ wave generation section. The individual sections are described as follows.

\section{A. MWPF based tunable OEO}

A light source from a distributed feedback (DFB) laser with a centre wavelength of $1553.73 \mathrm{~nm}$ and linewidth of $10 \mathrm{kHz}$ is routed via a 50:50 optical coupler to an OEO loop, and an OFCG. In the OEO loop, a PM serves two functions: (i) as an electrical-to-optical (E/O) converter, and (ii) as a MWPF when combined with a tunable optical bandpass filter (TOBF) [15]. When the PM is modulated with an external RF carrier, it generates optical sidebands with opposite phase around the optical carrier. If an optical bandpass filter with a center frequency equal to the optical carrier frequency is placed after the PM, then the anti-phased sidebands are symmetrically transmitted as shown in Fig. 2(a). If they beat immediately in a photo-detector a zero response is generated. However, if the (a)

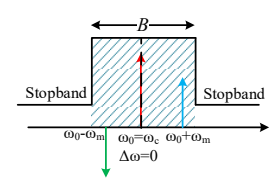

(c)
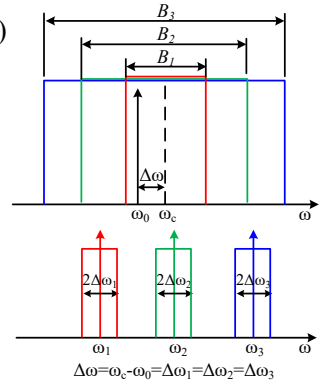

Centre frequency can be tuned from $\omega_{1}$ to $\omega_{3}$ by changing optical filter bandwidth from $B_{1}$ to $B$ (b)

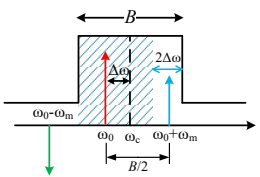

(d) $\quad B=B_{1}=B_{2}=B_{3}$

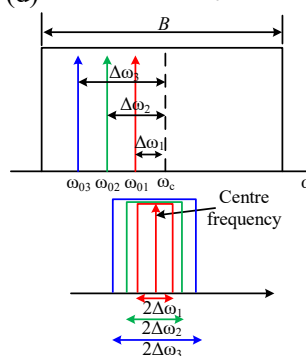

Bandwidth can be tuned from $2 \Delta \omega_{1}$ to $2 \Delta \omega_{3}$ by changing laser oscillation frequency from $\omega_{01}$ to $\omega_{03}$
Fig. 2. (a) Symmetrically transmitted sidebands (b) Asymmetrically transmitted sidebands (c) Centre frequency tuning by changing the optical bandwidth $(B)$ of the TOBF, and (d) Bandwidth tuning by changing the laser wavelength. centre frequency of the optical carrier $\left(\omega_{0}\right)$ is slightly shifted from the centre frequency of the optical filter $\left(\omega_{\mathrm{c}}\right)$, this gives asymmetrical sidebands suppression as shown in Fig. 2(b). The beating between the remaining of the unbalanced sidebands and the optical carrier will only be detected by the photodiode. This will generate a single sideband RF response, where the centre frequency and the bandwidth are determined by the spectral response of TOBF. The centre frequency in the electrical domain is equal to the half of the bandwidth of TOBF $(B / 2)$ and the bandwidth in electrical domain is equal to the twice of the offset between laser oscillation frequency and TOBF centre frequency $\left(\Delta \omega=\omega_{\mathrm{c}}-\omega_{0}\right)$, as shown in Fig 2(b). Tunability can be obtained by changing the wavelength, and bandwidth of TOBF. By changing the bandwidth of the TOBF, the centre frequency of the RF filter changes (Fig. 2(c)), while the bandwidth of the RF filter changes with the changes of offset frequency (Fig. 2(d)). A Yenista Optics XTM-50 (with a minimum and maximum bandwidth of $6.25 \mathrm{GHz}$, and $120 \mathrm{GHz}$, respectively) is employed as the TOBF, with a 99:1 optical coupler being used to monitor the optical spectrum after the TOBF. A dual loop balanced detection configuration is used in the feedback loop of the OEO, with short and long fiber lengths of $L_{1}=200$ $\mathrm{m}$ and $L_{2}=2 \mathrm{~km}$, respectively, which is fed into a balanced photodiode (DSC730, with a 3-dB bandwidth of $25 \mathrm{GHz}$, and responsivity of $0.60 \mathrm{~A} / \mathrm{W})$. Two electrical amplifiers are cascaded to compensate for the $\mathrm{E} / \mathrm{O}$ and $\mathrm{O} / \mathrm{E}$ conversion losses, and meet the loop gain condition for the OEO. The output of this amplifier chain is then fed back to the PM via a 50:50 electrical coupler, while the other coupler output is connected to $10: 90$ coupler. $10 \%$ of the output is used as monitoring in electrical spectrum analyzer, and $90 \%$ is used to drive the DDMZM (model PSI-3600D1) for optical comb generation.

\section{B. OFC generation}

The DFB laser is injected into a DD-MZM after being split by a 50:50 coupler and polarization controlled by a polarization controller (PC) as shown in Fig. 1. The optical frequency comb is generated by driving the two RF ports of the DD-MZM with RF signal of different amplitudes and phases [16]. The generated OEO electrical signal is further split into two signals, electrically amplified, and then connected to the two RF ports of the DD-MZM. Prior to amplification, the RF signal of one arm is attenuated while the other arm is phase shifted in order to satisfy the flat spectrum condition [16]. The output optical signal is optically amplified and split for monitoring by using an optical spectrum analyser, and the other output used for $\mathrm{THz}$ signal generation in the next section. 

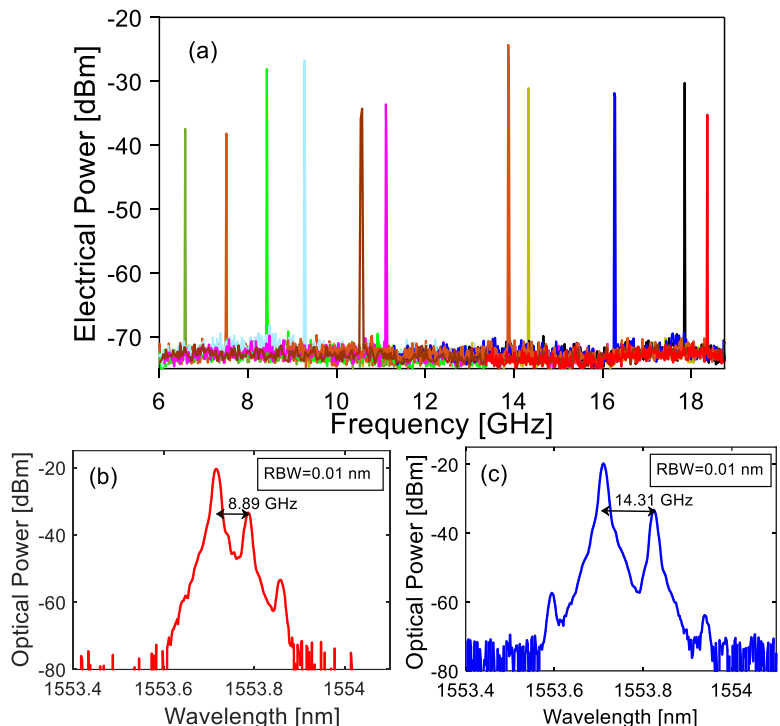

Fig. 3. (a) Electrical spectra of the generated microwave signal at different frequencies at point $\mathbf{B}$ of Fig.1; (b) and (c) optical spectra at the output of the TOBF for the oscillating frequency $8.89 \mathrm{GHz}$, and $14.31 \mathrm{GHz}$, respectively at point $\mathbf{A}$ of Fig.1.

\section{Optical two tone selection, and THz wave generation}

The generated optical comb after amplification is passed through a programmable wavelength selective switch (WSS) from Finisar to select two optical tones with the desired wavelength spacing. Then, the output signal is amplified, and filtered to remove the amplified spontaneous emission (ASE) noise before heterodyning at the UTC-PD. The UTC-PD is unpackaged device with an integrated coplanar waveguide (CPW). The output of the photodiode is emitted through a $20 \mathrm{dBi}$ horn antenna (WR-5.1) using a coplanar mm-wave probe. Another identical horn antenna is placed to receive the transmitted $\mathrm{THz}$ signal. In order to measure the phase noise of the received $\mathrm{THz}$ signal, we down-converted it to a microwave IF frequency within the range of RF components by using a sub-harmonic mixer (SHM). The local oscillator (LO) from Rohde\&Schwarz (SMA100A) is electrically multiplied $(\times 6)$ and mixed with $\mathrm{THz}$ signal in the SHM. The IF signal is then amplified and connected to an electrical spectrum analyser to measure the phase noise performance.

\section{EXPERIMENTAL RESULTS AND DISCUSSION}

As described earlier, the PM-TOBF combination functions as a tunable MWP filter. By tuning the bandwidth of the TOBF

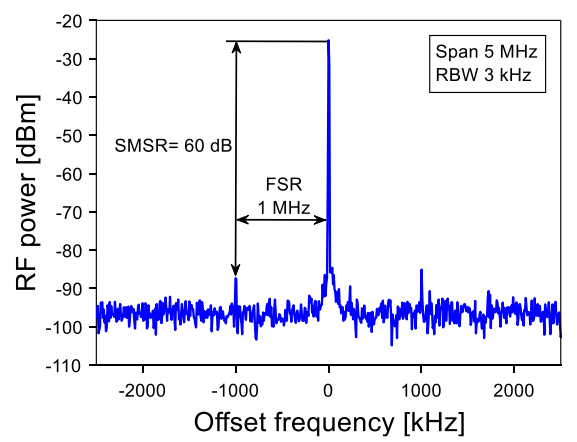

Fig.4. Electrical spectrum of the generated RF signal from $\mathrm{OEO}$ at $17.33 \mathrm{GHz}$ signal with span of $5 \mathrm{MHz}$ and RBW of $3 \mathrm{kHz}$ (measured at point $\mathbf{B}$ of Fig.1).

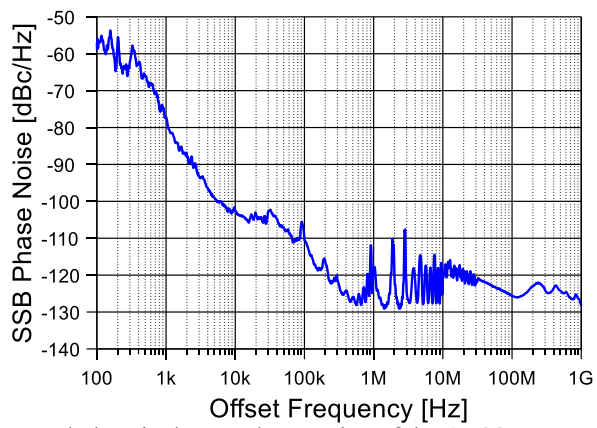

Fig. 5. Measured electrical SSB phase noise of the $17.33 \mathrm{GHz}$ oscillation.

filter, we were able to generate microwave signals from 6.58 $\mathrm{GHz}-18.36 \mathrm{GHz}$ - typical spectra of the generated RF carriers measured at a point $\mathbf{B}$ of Fig.1 are shown in Fig.3 (a). The optical spectrum after the TOBF is measured at point $\mathbf{A}$ in Fig. 1 with an optical spectrum analyzer of the resolution bandwidth of $0.01 \mathrm{~nm}$ and is depicted in Fig.3 (b) and Fig.3 (c) for 8.89 $\mathrm{GHz}$, and $14.31 \mathrm{GHz}$, respectively.

For $\mathrm{THz}$ signal generation, the OEO oscillation frequency was set to $17.33 \mathrm{GHz}$ by adjusting the bandwidth of the TOBF. The electrical spectrum of the generated signal was then measured from the $10 \%$ electrical splitter (at point $\mathbf{B}$ in Fig. 1) and is shown in Fig. 4. Side modes at an offset frequency of $1 \mathrm{MHz}$ from the carrier was observed at $60 \mathrm{~dB}$ suppression, and these correspond to the short fiber length $\left(L_{1}=200 \mathrm{~m}\right)$. The single side band (SSB) phase noise of the generated $17.33 \mathrm{GHz}$ signal was also measured. The phase noise level of $-103 \mathrm{dBc} / \mathrm{Hz}$ was found at $10 \mathrm{kHz}$ offset frequency as shown in Fig. 5.
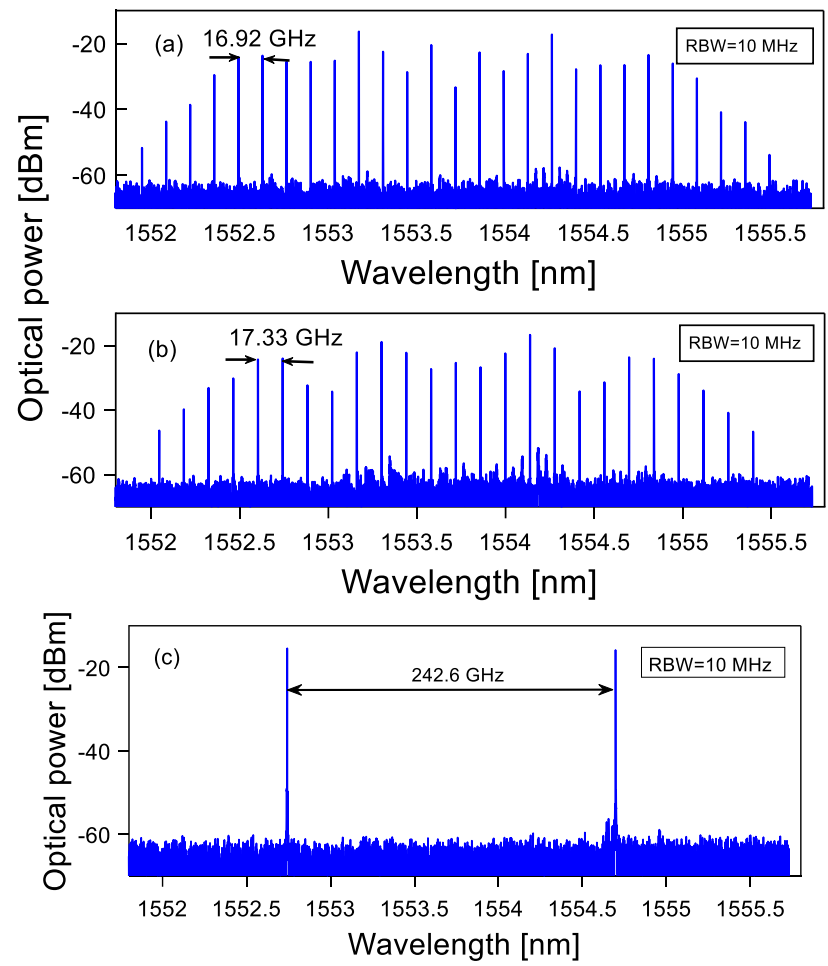

Fig.6. Optical spectra of the generated optical frequency combs with a frequency spacing of (a) $16.92 \mathrm{GHz}$ and (b) $17.33 \mathrm{GHz}$ at the center wavelength of $1553.73 \mathrm{~nm}$ (measured with an OSA at point $\mathbf{C}$ in Fig.1; (c) Optical spectrum of the selected comb lines (measured with an OSA at point $\mathbf{D}$ in Fig. 1). 


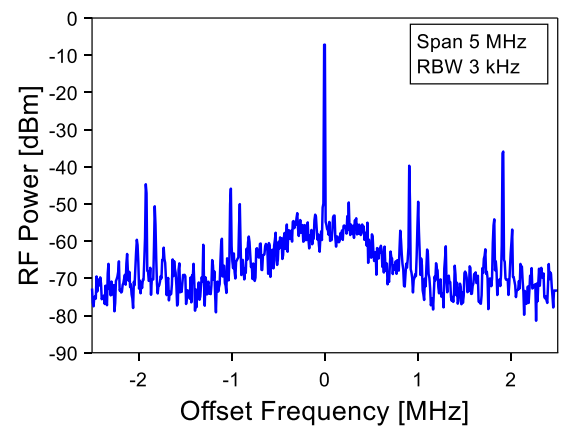

Fig.7. Electrical spectrum of the generated $242.6 \mathrm{GHz}$ signal after downconversion, measured at point $\mathbf{E}$ of Fig.1.

The optical comb signal for two different OEO oscillation frequency of $16.92 \mathrm{GHz}$, and $17.33 \mathrm{GHz}$ are shown in Fig. 6 (a) \& (b). A spectrum of 22 optical tones with a spacing of $17.33 \mathrm{GHz}$ are observed (Fig. 6(b)), covering a bandwidth of $360 \mathrm{GHz}$ within a $20 \mathrm{~dB}$ envelope. By selecting two optical comb lines, it is possible in principle to generate $\mathrm{THz}$ signals up to $360 \mathrm{GHz}$ through heterodyne detection at UTC-PD. Using a programmable WSS, we selected two optical comb lines separated by 14 lines to generate a $\mathrm{THz}$ signal at 242.6 GHz. Fig. 6(c) shows the optical spectra of the selected optical tones. The electrical spectra of the down-converted signals are shown in Fig. 7. We found $\sim 1 \mathrm{MHz}$ spaced side modes (corresponds to short length $L_{1}=200 \mathrm{~m}$ ) around the carrier and $\sim 100 \mathrm{kHz}$ spaced side modes (corresponds to the long length $\underline{L}_{2}=2 \mathrm{~km}$ ) with relatively low amplitude around the $\sim 1 \mathrm{MHz}$ spaced side modes. The SSB phase noise of the downconverted signals were measured as shown in Fig. 8. The SSB phase noise is $-78 \mathrm{dBc} / \mathrm{Hz}$ at $10 \mathrm{kHz}$ offset frequency which is degraded by $25 \mathrm{~dB}$. This degradation also includes the phase noise addition during the down-conversion process coming from optical and electrical amplifiers. Although the SSB phase noise of our OEO is comparable to other reported designs, the SSB phase noise of the system could be improved by using a long fiber. In addition, the long-term stability of the set-up could be improved by thermal stabilization. The refractive index of the fiber depends on temperature, hence temperature variation results in frequency drift of the OEO oscillation. This oscillation frequency drift could be controlled by using a temperature stabilized box or by phase locking with an external reference. Recently, hollow core photonic band gap (HC-PBG) fiber have been considered in OEO implementations to enhance long term stability, and improve the temperature stability by a factor of more than 15 , as compared to standard single mode fiber [17].

\section{CONCLUSION}

We have demonstrated a low phase noise $\mathrm{THz}$ generation technique in which a tunable $\mathrm{OEO}$ is used to drive an $\mathrm{OFC}$ generator. The demonstrated system can generate a tunable $\mathrm{THz}$ signal with low phase noise by tuning the OEO oscillation frequency. We generated $\mathrm{THz}$ signal at $242.6 \mathrm{GHz}$ with a phase noise level of $-78 \mathrm{dBc} / \mathrm{Hz}$ at $10 \mathrm{kHz}$ offset when the OEO oscillation frequency was $17.33 \mathrm{GHz}$. The system has the potential to apply in multi-carrier or multi-band radio over fiber

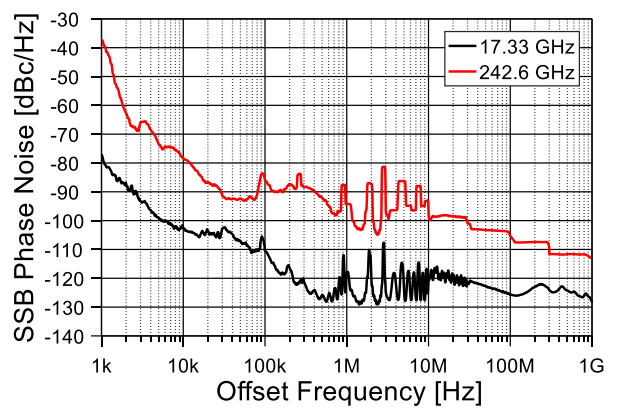

Fig.8. SSB phase noise of the generated $242.6 \mathrm{GHz}$ signal (after downconversion)

link with reconfigurable $\mathrm{THz}$ carrier frequency. The signal stability of generated $\mathrm{THz}$ signal depends on the OEO stability.

\section{REFERENCES}

[1] S. Iezekiel, Microwave photonics: devices and applications. Wiley, 2009.

[2] H. Shams et al., "Coherent frequency tuneable thz wireless signal generation using an optical phase lock loop system," in MWP 2017 2017 International Topical Meeting on Microwave Photonics, 2017, vol. 2017-Decem, pp. 2-5.

[3] S. Koenig et al., "Wireless sub-THz communication system with high data rate," Nat. Photonics, vol. 7, pp. 977-981, 2013.

[4] D. Hillerkuss et al., "High-quality optical frequency comb by spectral slicing of spectra broadened by SPM," IEEE Photonics J., vol. 5, no. 5, 2013.

[5] X. S. Yao and L. Maleki, "Optoelectronic microwave oscillator," $J$. Opt. Soc. Am. B, vol. 13, no. 8, p. 1725, 1996.

[6] L. Maleki, "The opto-electronic oscillator (OEO): Review and recent progress," 2012 Eur. Freq. Time Forum, pp. 497-500, 2012.

[7] D. Eliyahu, D. Seidel, and L. Maleki, "Phase noise of a high performance $\mathrm{OEO}$ and an ultra low noise floor cross-correlation microwave photonic homodyne system," in 2008 IEEE International Frequency Control Symposium, FCS, 2008, pp. 811-814.

[8] X. S. Yao and L. Maleki, "Multiloop optoelectronic oscillator," IEEE J. Quantum Electron., vol. 36, no. 1, pp. 79-84, 2000.

[9] H. Peng et al., "Tunable DC-60 GHz RF generation utilizing a dualloop optoelectronic oscillator based on stimulated brillouin scattering," J. Light. Technol., vol. 33, no. 13, pp. 2707-2715, 2015.

[10] W. Li and J. Yao, "A wideband frequency tunable optoelectronic oscillator incorporating a tunable microwave photonic filter based on phase-modulation to intensity-modulation conversion using a phaseshifted fiber bragg grating," IEEE Trans. Microw. Theory Tech., vol. 60, no. 6 PART 1, pp. 1735-1742, 2012.

[11] X. Xie et al., "Wideband tunable optoelectronic oscillator based on a phase modulator and a tunable optical filter.," Opt. Lett., vol. 38, no. 5, pp. $655-7,2013$.

[12] X. Xie et al., "Widely Tunable Dual-loop Optoelectronic Oscillator," Cleo 2014, no. c, p. STu2G.2, 2014.

[13] G. K. M. Hasanuzzaman, A. Kanno, P. T. Dat, and S. Iezekiel, "Wband radio-over-fiber link based on self-oscillating optical frequency comb generator," Opt. Fiber Commun. Conf., vol. Part F84-O, pp. 5-7, 2018.

[14] A. Kanno, G. K. M. Hasanuzzaman, N. Yamamoto, and S. Iezekiel, "Optical frequency comb applied optoelectronic oscillator for millimeter-wave signal generation and its application," in presented at SPIE Defense and Security, Passive and Active Millimeter-Wave Imaging XXI, 2018, vol. 10634, p. paper 10634-13.

[15] T. Chen, X. Yi, L. Li, and R. Minasian, "Single passband microwave photonic filter with wideband tunability and adjustable bandwidth.," Opt. Lett., vol. 37, no. 22, pp. 4699-701, 2012.

[16] T. Sakamoto, T. Kawanishi, and M. Izutsu, "Asymptotic formalism for ultraflat optical frequency comb generation using a Mach-Zehnder modulator.," Opt. Lett., vol. 32, no. 11, pp. 1515-1517, 2007.

[17] U. S. Mutugala et al., "Optoelectronic oscillator incorporating hollowcore photonic bandgap fiber," Opt. Lett., vol. 42, no. 13, pp. $2647-$ 2650, 2017. 
